Topics

\title{
Computational Analysis of Aerodynamic Performance of Mars Airplane
}

\author{
By Naoya FusIOKA ${ }^{1)}$, Taku NONOMURA ${ }^{2)}$, Akira OYAmA ${ }^{2)}$, Makoto YAMAмOTO ${ }^{1)}$ and Kozo FuJII $^{2)}$ \\ ${ }^{1)}$ Department of Mechanical Engineering, Tokyo University of Science, Tokyo, Japan \\ ${ }^{2)}$ Institute of Space and Astronautical Science, Japan Aerospace Exploration Agency, Sagamihara, Japan
}

(Received June 28th, 2013)

\begin{abstract}
Flow field and aerodynamic performance of the Mars airplane with a complete aircraft configuration are analyzed by RANS simulations. At the Reynolds number of $3.3 \times 10^{4}$, a flow field is solved by an unstructured three-dimensional compressible CFD solver (LS-FLOW). Here, the Mars airplane is assumed to have the Ishii airfoil as the main wing shape. The Ishii airfoil is known as its good performance at the low Reynolds number condition. An objective of the present study is to clarify flow structures around a complete aircraft, for optimization of design of the Mars airplane. The results show that the features of the aerodynamic coefficients correspond to those of experimental results and the contribution of the main wing is significant on the aerodynamic characteristics of the entire airplane.
\end{abstract}

Key Words: Aerodynamic Performance, CFD, Low Reynolds Number, Mars Exploration

$\begin{array}{cl}\text { Nomenclature } \\ b & : \text { half span of main wing } \\ c & : \text { chord of main wing } \\ C_{D} & : \text { drag coefficient } \\ c_{l} & : \text { sectional lift coefficient } \\ C_{L} & : \text { lift coefficient } \\ C_{p} & : \text { pressure distribution } \\ L / D & : \text { lift-to-drag ratio } \\ \operatorname{Re} & : \text { Reynolds number } \\ \alpha & : \text { angle of attack }\end{array}$

\section{Introduction}

Current and previous missions to Mars have been conducted by ground-based rovers and orbiters. Rovers provide detailed data on the surface but their reach is limited to a small area. On the other hand, orbiting sensors provide spatially wide data but their resolution is very low. There is no approach which has the intermediate role between rovers and orbiters. Thus, the idea of Mars exploration by an airplane has been discussed in JAXA/ISAS for several years.

An airplane design for Mars is very different from the conventional airplanes on Earth. First, the Reynolds number of the flow condition for the Mars airplane is smaller $\left(\operatorname{Re}=10^{5}\right)$ than the typical airplanes on Earth $\left(R e=10^{7}\right)$ because the atmosphere on Mars is much thinner than that on Earth. Second, its cruising Mach number becomes high because the speed of sound on Mars is smaller than that on Earth and the aircraft needs a certain cruising speed to gain sufficient dynamic pressure for lift in the thin atmosphere. Because aircrafts flying in such a flow condition on Earth are limited to high-altitude airplanes, the optimum design of the Mars airplane is not well known.
Kojima et $\mathrm{al}^{1)}$ have showed that thin airfoils are appropriate at the low Reynolds number condition. The Ishii airfoil is one of high performance airfoils. Figure 1 shows the shape of the Ishii airfoil. The Ishii airfoil has several prominent features: (i) the leading edge is round, (ii) the upper surface of airfoil is flat, (iii) the lower surface of airfoil has cambered shape. Two features (ii) and (iii) correspond to the proposition of $\mathrm{Schmitz}^{2)}$ for low Reynolds number. Anyoji et $\mathrm{al}^{3,4)}$ analyzed the aerodynamic performance of the Ishii airfoil using large eddy simulations (LES). The results show that the Ishii airfoil has a high lift-to-drag ratio in wide range of the angle of attack. Thus, the Ishii airfoil is expected to be a good candidate as the main wing of the Mars airplane.

When an airplane is designed, not only an airfoil itself but also a wing-body configuration should be optimized. Thus, several studies have been conducted to understand effects of a wing-body configuration on its aerodynamic characteristics. However, those studies mainly focus on the high Reynolds number condition, and such effects under the low Reynolds number condition have not been discussed well. The flow field includes several factors, such as a laminar separation bubble, a wing-tip vortex, a wing-body interference and others. Therefore, the flow field might be more complicated than the high Reynolds number condition. The optimum wing-body configuration should be considered and the aerodynamic characteristic should be improved.

In the present study, the flow field with a complete aircraft configuration for the Mars airplane is simulated by Reynolds Averaged Navier-Stokes (RANS) simulations. The simulation result clarifies aerodynamic performance of the current Mars airplane design. An objective of the present study is to evaluate aerodynamic performances of the Mars airplane with varying the angles of attack. 


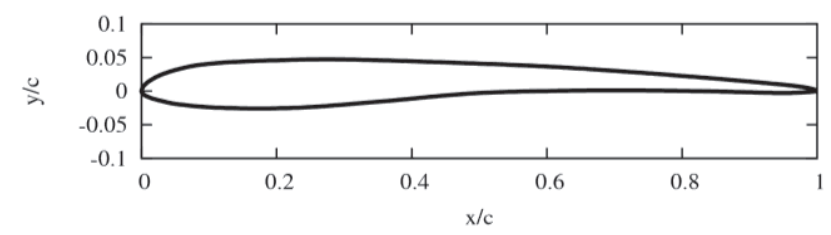

Fig. 1. Shape of Ishii airfoil.

\section{Flow Conditions}

For the simulation to obtain the aerodynamic coefficient, the angle of attack $(\alpha)$ is changed from 0 to $12 \mathrm{deg}$ at intervals of $2 \mathrm{deg}$. The Reynolds number (Re) based on the chord length and free stream velocity is set to $3.3 \times 10^{4}$ to compare with the experimental result. ${ }^{5)}$ The Mach number is set to 0.2 to ignore the compressibility. Reference 6) shows an effect of the specific heat ratio is negligible in the present Mach number region. Thus, the specific heat ratio is set to 1.4. These conditions are summarized in Table 1.

Figure 2 shows the analysis object. Its dimension is based on the scale model of the Mars airplane used for the experiment. ${ }^{5)}$ The real Mars airplane has some propellers for a propulsion system. For simplicity, propellers are abbreviated. The shape of the main wing is the Ishii airfoil with aspect ratio of 5.2. NACA0009 is used as the tail wing. The incident angle of both the main and tail wing is $0 \mathrm{deg}$.

\begin{tabular}{|c|c|}
\multicolumn{1}{c}{ Table 1. $\quad$ Flow conditions. } \\
\hline Reynolds number & $3.3 \times 10^{4}$ \\
\hline Mach number & 0.2 \\
\hline Specific heat ratio & 1.4 \\
\hline
\end{tabular}

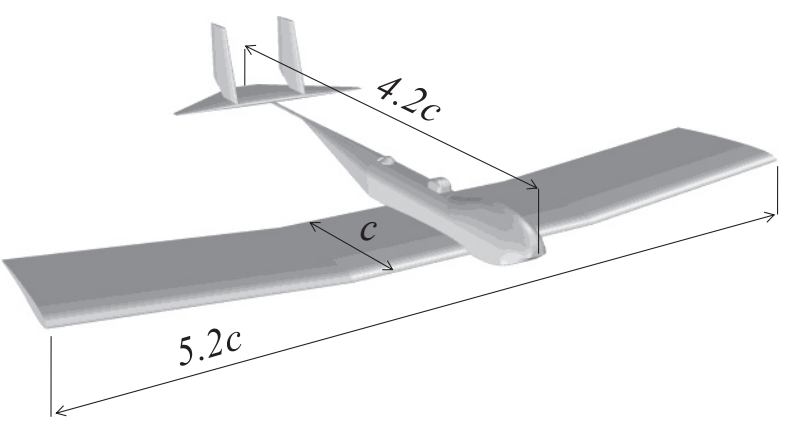

Fig. 2. Analysis object.

\section{Numerical Method}

\subsection{Flow solver}

The computational code employed in this study is LS-FLOW: a JAXA's in-house, compressible Navier-Stokes solver for arbitrary polygons. Numerical fluxes for convective terms are evaluated by the SLAU. ${ }^{7)}$ The flow field is assumed to be fully turbulent, and the Spalart-Allmaras turbulence mode $l^{8)}$ is applied. Time integration is conducted by using the LU-SGS implicit method. ${ }^{9)}$

\subsection{Computational grid}

An unstructured grid generator called MEGG3D ${ }^{10)}$ is employed for generation of a grid. Here, MEGG3D is able to generate hybrid grids that consist of elements of tetrahedral, pyramid and prism types.
Figure 3 shows the grid with a complete aircraft configuration for the RANS computation. The grid has approximately 2 million nodes and 6 million elements. Near a surface, an element type is selected to be a prism type. The first element away from the surface is set to be $0.05 c / \sqrt{R e}$. The distance from the body surface to the computational outer boundary is $20 c$.

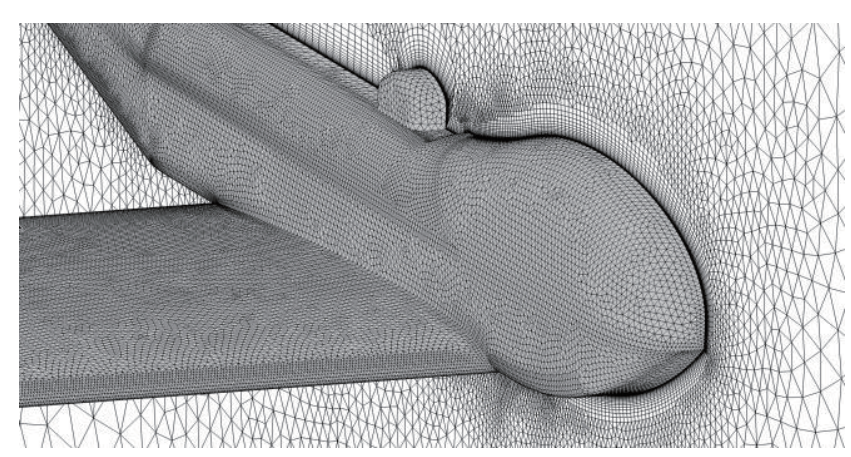

Fig. 3. Computational grid.

\section{Results and Discussions}

\subsection{Aerodynamic performances}

The aerodynamic force coefficients as functions of $\alpha$ are plotted in Fig. 4. Computational results are compared with the experimental results. ${ }^{5)}$ In the two-dimensional airfoil flow simulations performed by LES, ${ }^{3,4)}$ the nonlinear lift curve is observed around $\alpha=4 \mathrm{deg}$. However, the lift curve has a linearity at $\alpha=0$ to $6 \mathrm{deg}$ in a complete aircraft configuration. The experimental result also shows that the lift curve is linear. Therefore, the current design of the Mars airplane has the linear lift coefficient for a low angle of attack regime. For more than $\alpha=8 \mathrm{deg}$, the lift coefficient is almost constant. The drag coefficient curve has a similar trend to the experimental result.

Although the computational result is slightly different compared with the experimental result, the computational results show a similar trend to the experimental results. Thus, the simulation is assumed to capture the dominant feature of flow structure.

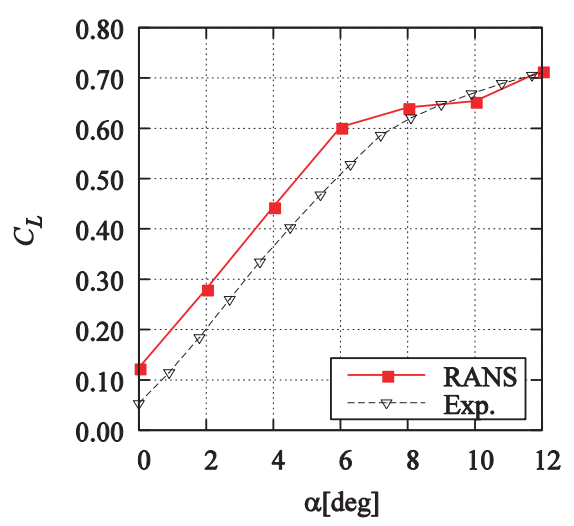

(a) Lift coefficient $\left(C_{L}\right)$ 


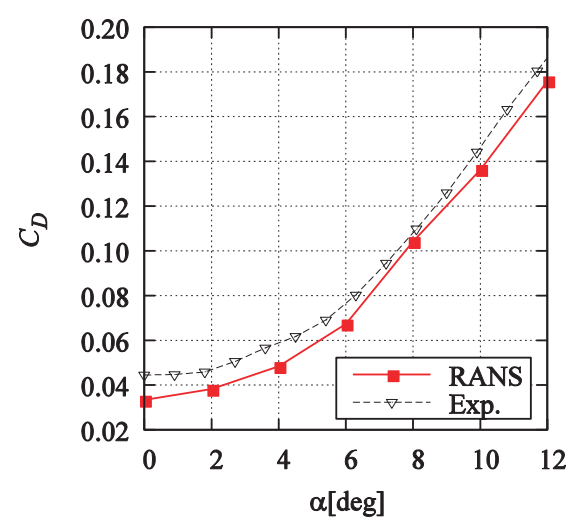

(b) Drag coefficient $\left(C_{D}\right)$

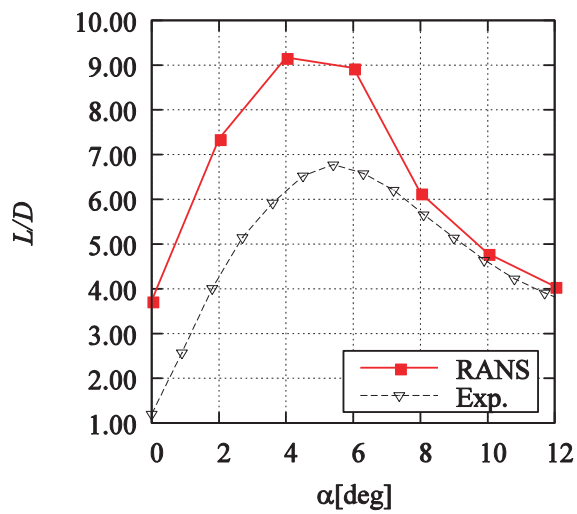

(c) Lift-to-drag ratio $(L / D)$

Fig. 4. Aerodynamic coefficients as function of $\alpha$.

\subsection{Decomposition analysis}

Figure 5 shows the half body of the Mars airplane from top view. The surface of the Mars airplane is divided into four panels. In Fig.5, Others represent all the additional objects on the Mars airplane such as sensors. Figure 6 shows the contribution of each panel to lift and drag coefficients, and Table 2 indicates the percentage of the contribution. The lift contribution of the main wing is more than $90 \%$ for almost all $\alpha$. The lift coefficient of the Mars airplane strongly depends on the main wing. The drag contribution of the main wing is more than $60 \%$ and it is the most dominant element for all $\alpha$. Thus, reducing drag of the main wing improves the aerodynamic performance of the Mars airplane. Other panels are almost constant with varying $\alpha$ compared with the drag contribution of the main wing. At $\alpha=6 \mathrm{deg}$ (steady flight angle), the drag contribution of all panels excluding the main wing is approximately $24 \%$. Among them, the contributions of the body and tail wing are large. In order to improve the gliding performance, it is necessary to design a low drag body and a tail wing.

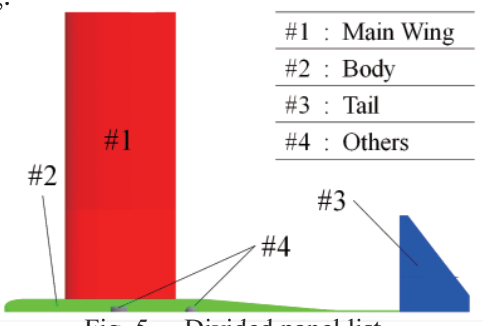

Fig. 5. Divided panel list.

\begin{tabular}{|c|c|}
\hline \begin{tabular}{ll}
$\longrightarrow$ & Total \\
\hdashline & MainWing \\
&
\end{tabular} & 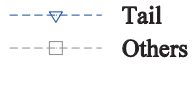 \\
\hline
\end{tabular}

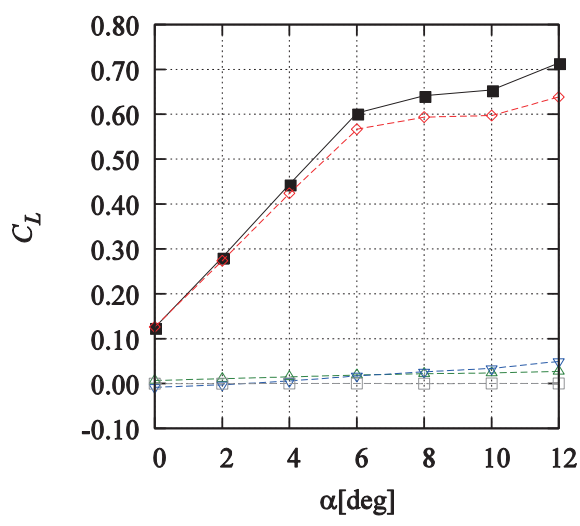

(a) Lift coefficient

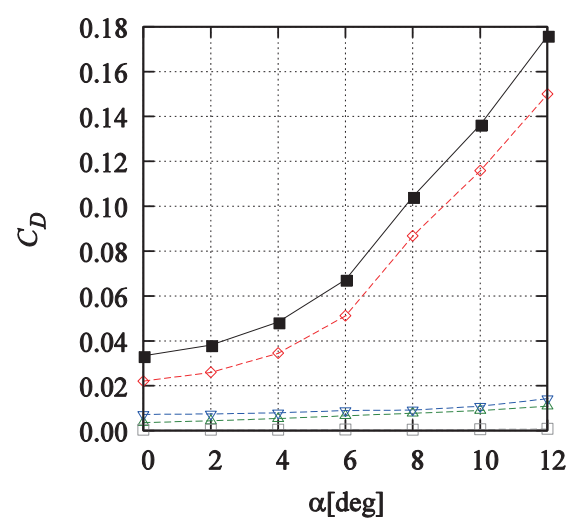

(b) Drag coefficient

Fig. 6. Each panel contribution to aerodynamic coefficients.

Table 2. Component ratio of aerodynamic coefficient.

(a) Lift coefficient [\%]

\begin{tabular}{|c|c|c|c|c|}
\hline$\alpha[$ deg.] & Main Wing & Body & Tail & Others \\
\hline 0 & 101.43 & 5.12 & -6.81 & 0.26 \\
\hline 2 & 97.21 & 3.60 & -1.02 & 0.21 \\
\hline 4 & 95.40 & 3.13 & 1.29 & 0.18 \\
\hline 6 & 94.05 & 3.01 & 2.78 & 0.17 \\
\hline 8 & 92.55 & 3.27 & 4.02 & 0.16 \\
\hline 10 & 91.28 & 3.47 & 5.06 & 0.19 \\
\hline 12 & 89.32 & 3.60 & 6.83 & 0.25 \\
\hline
\end{tabular}

(b) Drag coefficient [\%]

\begin{tabular}{|c|c|c|c|c|}
\hline$\alpha$ [deg.] & Main Wing & Body & Tail & Others \\
\hline 0 & 66.31 & 9.46 & 21.98 & 2.25 \\
\hline 2 & 67.99 & 9.88 & 19.58 & 2.55 \\
\hline 4 & 71.38 & 9.58 & 16.58 & 2.46 \\
\hline 6 & 76.03 & 8.59 & 13.36 & 2.03 \\
\hline 8 & 83.31 & 6.61 & 8.82 & 1.26 \\
\hline 10 & 84.96 & 5.89 & 8.01 & 1.13 \\
\hline 12 & 85.20 & 5.64 & 8.11 & 1.05 \\
\hline
\end{tabular}

\subsection{Time averaged flow fields}

Figure 7 shows an oil-flow image of the main wing. At $\alpha=$ 0 deg, flow attaches on the upper surface, but flow separates and reattaches on the lower surface. At $\alpha=4$ to $6 \mathrm{deg}$, the separation and reattachment are observed on the upper surface. Flow near a wing-tip is affected by the wing-tip vortex. Especially at $\alpha=4 \mathrm{deg}$, the wing-tip vortex suppresses 


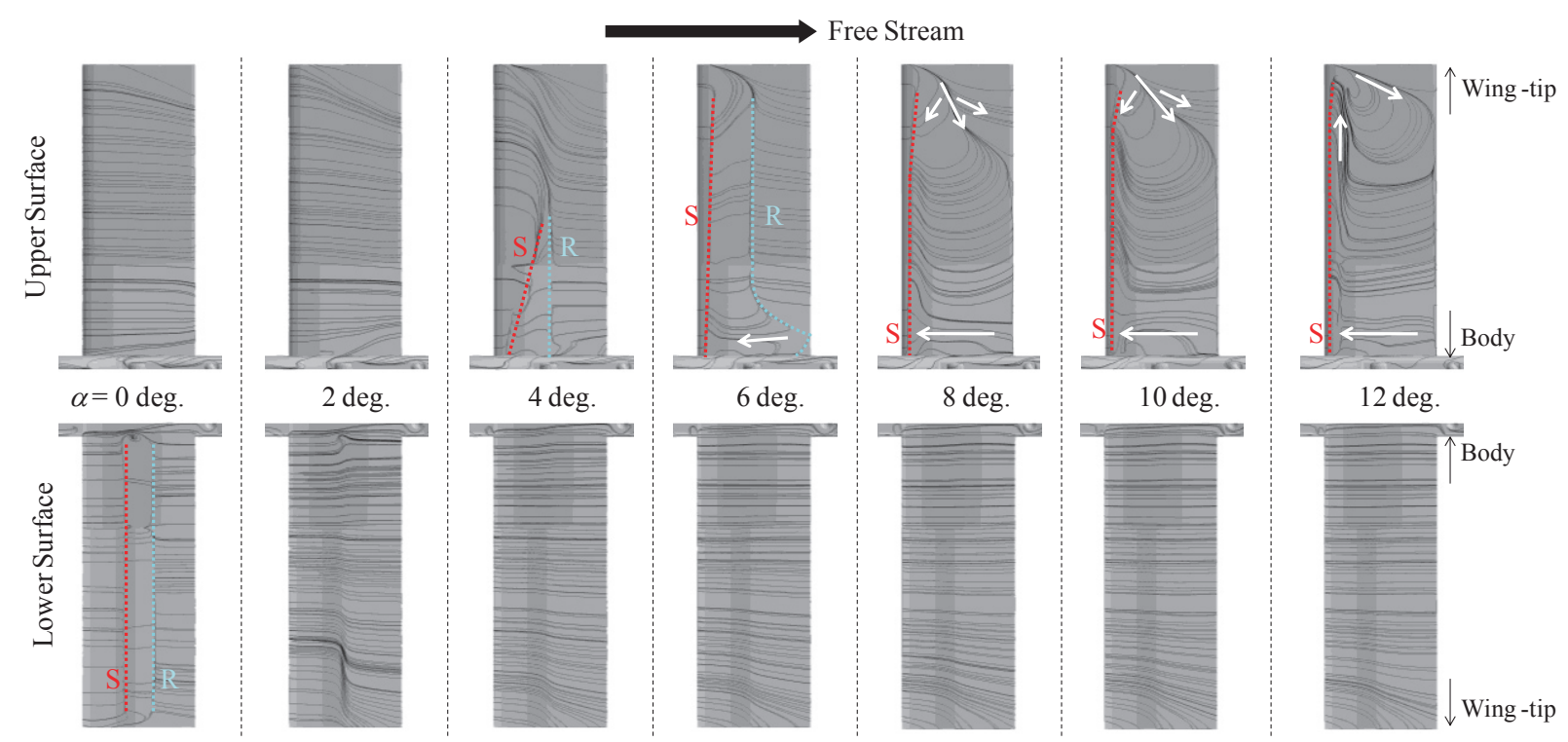

Fig. 7. Time averaged surface stream line on the main wing. The lines with notifications $\mathrm{S}$ and $\mathrm{R}$ indicate the locations of separation and reattachment. The white arrows indicate the flow direction near wall.

separation from near the wing-tip to mid-span. From $\alpha=8$ to $12 \mathrm{deg}$, the flow forms the massive separation from the leading edge. This separation and reattachment feature for $\alpha$ corresponds to those of the two-dimensional airfoil flow computed by LES.

\subsection{Three-dimensional effects on the main wing}

In order to evaluate the three-dimensional effects on the main wing, the pressure coefficient $\left(C_{p}\right)$ distributions on the wing section at $\alpha=6 \mathrm{deg}$ (steady flight angle) are plotted in Fig. 8. Figure 8 (a) shows the oil-flow image with the positions of 3 locations. Subsequent figures include the result of the two-dimensional airfoil flow plotted by dash line. Figure 8 (b) presenting $C_{p}$ close to the body shows the effects of the wing-body interference. The flow separates and reattaches at the trailing edge. The laminar separation bubble is longer than on mid-span. Figure 8 (c) presenting $C_{p}$ on the mid-span shows a similar trend to the two-dimensional airfoil flow, but the contribution to the lift coefficient from the laminar separation bubble decreases. In Figure 8 (d) presenting $C_{p}$ close to the wing-tip, the separation and reattachment are not observed and the integration of the pressure coefficient on a wing-tip side is greatly decreased by the wing-tip effect. The integration of the pressure coefficient is lower than that of the two-dimensional airfoil flow in all locations. It is noted in detail that the suction peak is suppressed and the positive pressure slightly decreases on the lower surface. Figure 9 shows the distribution of sectional lift coefficients $\left(c_{l}\right)$ as the function of normalized span position. Sectional lift coefficients are the integration of the pressure coefficient. It is clarified that the effect of the wing-tip is larger than that of the wing-body interference.

The result of the two-dimensional airfoil flow shows that the lift curve is nonlinear. When the flow regime changes from the attached flow to the separated flow, the lift coefficient is enhanced by the laminar separation bubble. It was reported that this transition of the flow regime makes the lift curve nonlinear. Although the same transition is observed as shown in Fig. 7, the lift curve is linear in a complete aircraft configuration. It is considered that the reason why the lift curve becomes linear is due to the three-dimensional effects. This might be because the flow change by generation of laminar separation becomes milder due to the wing-tip effects.

\section{Conclusions}

The aerodynamic performance of the Mars airplane with a complete aircraft configuration is analyzed by performing RANS simulations. The results clarify the outline of the flow field, how the main wing, the body, the tail wing and others affect to the aerodynamic performance.

The decomposition analysis shows the most dominant factor to determine the aerodynamic performance is the main wing. The main wing has a more than $90 \%$ contribution to the lift coefficient, and a more than $60 \%$ contribution to the drag coefficient. To improve the aerodynamic performance of the main wing is especially effective toward the optimum design of the Mars airplane. The other components contribute approximately $10 \%$ to the lift coefficient and 10 to $40 \%$ to the drag coefficient. Changing the shape of these components for reduction of drag raises the whole aerodynamic performance.

Three-dimensional effects such as the wing-body interference and the wing-tip effect affect the flow regime on the upper surface. These three-dimensional effects seem to be the reason why the lift curve becomes linear.

\section{Acknowledgments}

The Author's thank Mr. Junya Aono of Research Center of Computational Mechanics, Inc., Dr. Yasushi Ito of Japan Aerospace Exploration Agency and Mr. Hiroyuki Ito of Ryoyu Systems Co., Ltd. for valuable advice.

The present research was partially supported by a Grant-in-Aid for Scientific Research (24246136). 


\section{References}

1) Kojima, R., Nonomura, T.,Oyama, A.,Fujii, K. : Large Eddy Simulation of Low-Reynolds-Number Flow Over Thick and Thin NACA Airfoils, Journal of Aircraft, 50 No.1, (2013)

2) Schmitz, F. W. : Aerodyamics of the Model Airplane Part1, RSIC-721, 1967.

3) Anyoji, M., Nonomura, T.,Oyama, A.,Fujii, K., Nose, K.,Numata, D., Nagai, H.,Asai, K. : Aerodynamic Characteristics of Ishii Airfoil at Low Reynolds Numbers, OS6-11, 2011.

4) Aono, H., Anyoji, M., Nonomura, T.,Oyama, A.,Fujii, K. : A numerical study of the effects of airfoil shape on low Reynolds number aerodynamics, ECT.131, 2012.

5) Anyoji, M., Okamoto, M., Hidaka, H.,Oyama, A.,Fujii, K. : Aerodynamic Performance Evaluation of a Mars Airplane by Scale-model Wind Tunnel Tests (in Japanese), JSASS-2013-H012

6) Anyoji, M. : Development of a Mars Wind Tunnel and Its Applications to Low Reynolds Number and High-subsonic Airfoil Testing, Ph.D. thesis, Tohoku University, 2011

7) Shima, E., Kitamura, K. : On new simple low-dissipation scheme of AUSM-family for all speeds, AIAA Paper, 2009-0136, 2009.

8) Spalart, P. R. and Allmaras, S. R. : A One-Equation Turbulence Model for Aerodynamic Flows, AIAA Paper 92-0439, 1992.

9) J.M.Weiss, W. A. Smith : Preconditioning applied to variable and constant density flows,AIAA J., 33(11), 2050-2057, 1995.

10) Ito, Y., Alan, M. Shih, Bharat, K. Soni : Unstructured Mesh Generation Using MEGG3D - Mixed-Element Grid Generator in Three Dimensions, EPMESC, 2007.

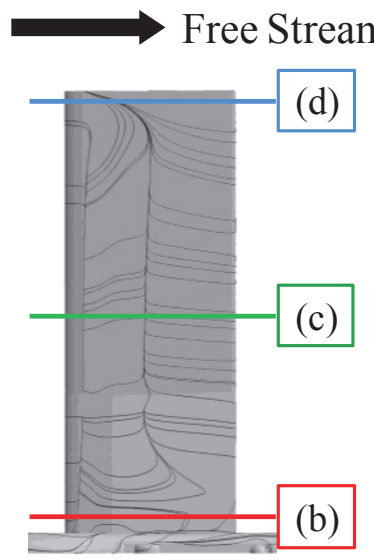

(a) Intended locations

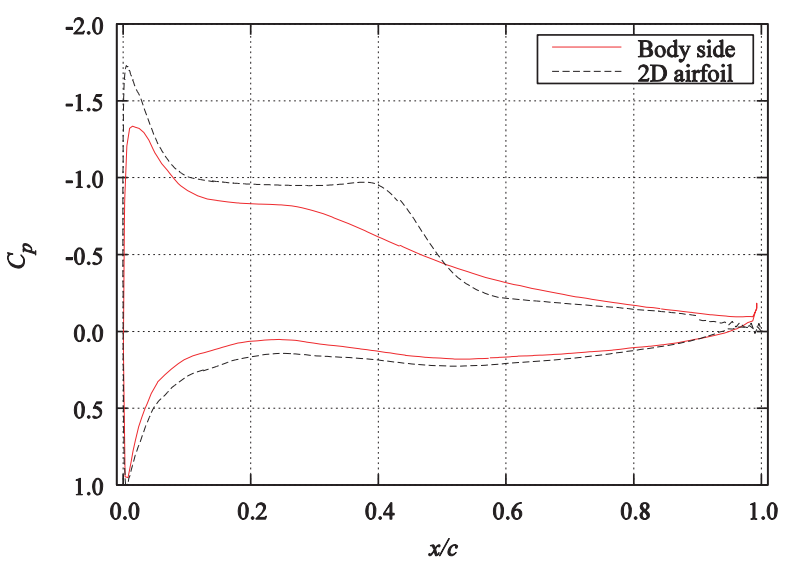

(b) Body side

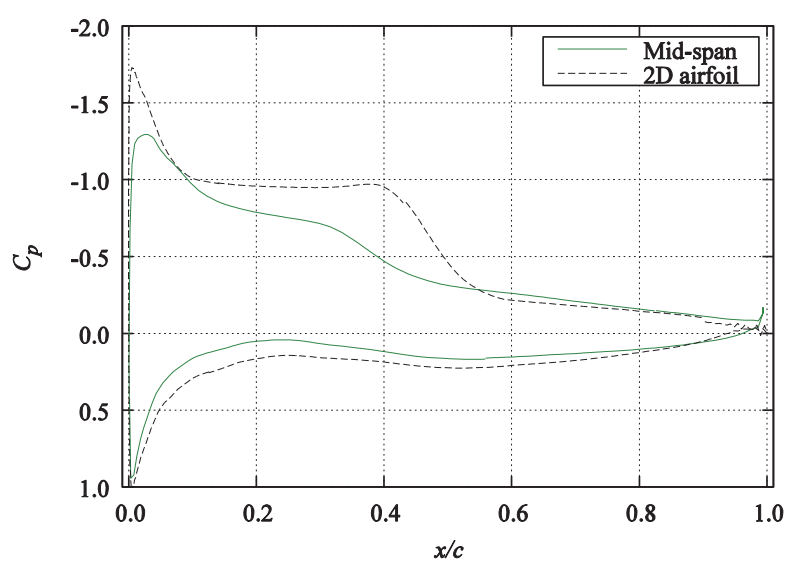

(c) Mid-span

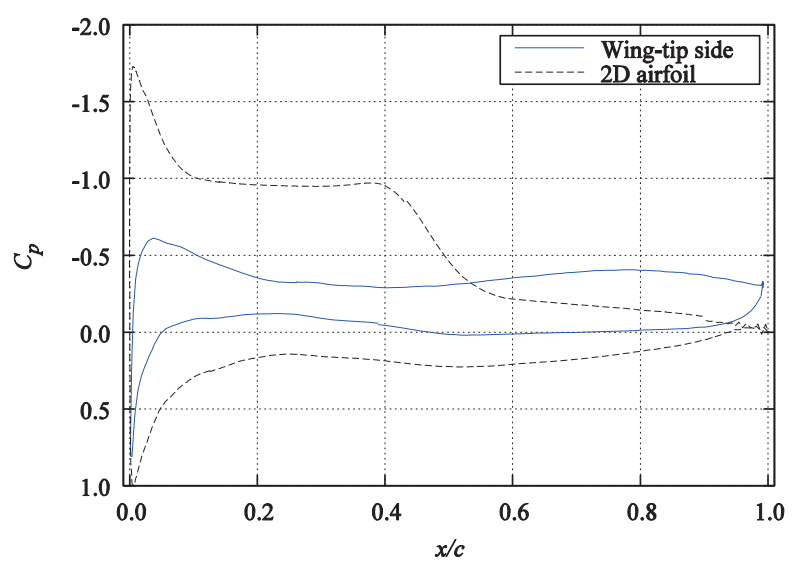

(d) Wing-tip side

Fig. 8. Pressure coefficient distributions at $\alpha=6 \mathrm{deg}$.
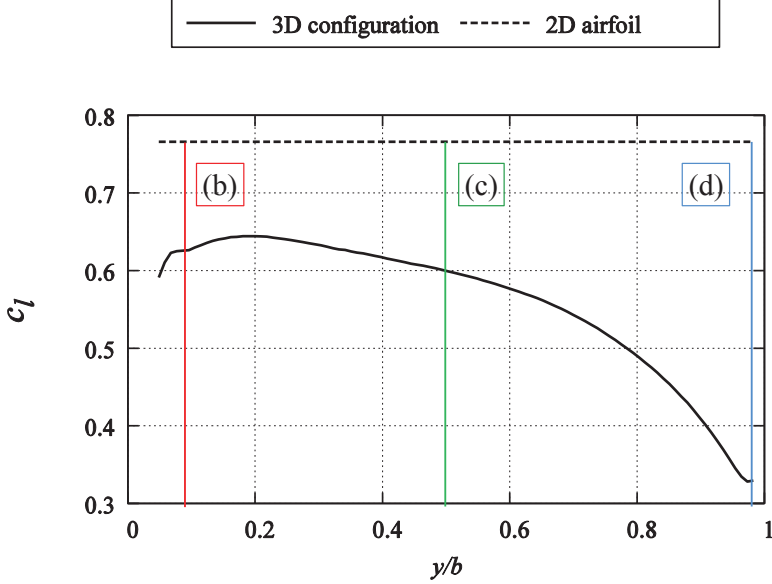

Fig. 9. Sectional lift coefficient in the span-direction. 